\title{
CaMKII inhibition rectifies arrhythmic phenotype in a patient-specific model of catecholaminergic polymorphic ventricular tachycardia
}

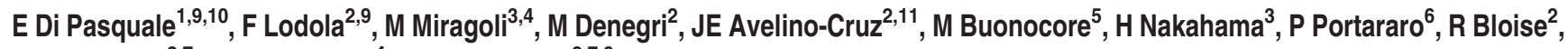 \\ C Napolitano ${ }^{2,7}$, G Condorelli $^{\star, 4}$ and SG Priori ${ }^{\star, 2,7,8}$
}

Induced pluripotent stem cells (iPSC) offer a unique opportunity for developmental studies, disease modeling and regenerative medicine approaches in humans. The aim of our study was to create an in vitro 'patient-specific cell-based system' that could facilitate the screening of new therapeutic molecules for the treatment of catecholaminergic polymorphic ventricular tachycardia (CPVT), an inherited form of fatal arrhythmia. Here, we report the development of a cardiac model of CPVT through the generation of iPSC from a CPVT patient carrying a heterozygous mutation in the cardiac ryanodine receptor gene (RyR2) and their subsequent differentiation into cardiomyocytes (CMs). Whole-cell patch-clamp and intracellular electrical recordings of spontaneously beating cells revealed the presence of delayed afterdepolarizations (DADs) in CPVT-CMs, both in resting conditions and after $\beta$-adrenergic stimulation, resembling the cardiac phenotype of the patients. Furthermore, treatment with KN-93 (2-[N-(2-hydroxyethyl)]- $N$-(4methoxybenzenesulfonyl)]amino- $N$-(4-chlorocinnamyl)- $N$-methylbenzylamine), an antiarrhythmic drug that inhibits $\mathrm{Ca}^{2+}$ /calmodulin-dependent serine-threonine protein kinase II (CaMKII), drastically reduced the presence of DADs in CVPT-CMs, rescuing the arrhythmic phenotype induced by catecholaminergic stress. In addition, intracellular calcium transient measurements on 3D beating clusters by fast resolution optical mapping showed that CPVT clusters developed multiple calcium transients, whereas in the wild-type clusters, only single initiations were detected. Such instability is aggravated in the presence of isoproterenol and is attenuated by KN-93. As seen in our RyR2 knock-in CPVT mice, the antiarrhythmic effect of KN-93 is confirmed in these human iPSC-derived cardiac cells, supporting the role of this in vitro system for drug screening and optimization of clinical treatment strategies.

Cell Death and Disease (2013) 4, e843; doi:10.1038/cddis.2013.369; published online 10 October 2013

Subject Category: Experimental Medicine

Induced pluripotent stem cell (iPSC) technology has been proposed as a valuable approach for studying the pathophysiology of human diseases in vitro. iPSCs are generated by the reprogramming of somatic cells through the expression of ectopic transcription factors, and have been shown to be able to differentiate into all cell types of the body, including functional cardiomyocytes (CMs). ${ }^{1-3}$

\footnotetext{
${ }^{1}$ Istituto di Ricerca Genetica e Biomedica, National Research Council of Italy, Milan, Italy; ${ }^{2}$ Molecular Cardiology, IRCCS Fondazione Salvatore Maugeri, Pavia, Italy; ${ }^{3}$ Humanitas Clinical and Research Center, University of Milan, Rozzano (MI), Italy; ${ }^{4}$ Department of Bioscience, Center of Excellence for Toxicological Research INAIL exISPESL, University of Parma, Parma, Italy; ${ }^{5}$ Unit of Clinical Neurophysiology and Neurodiagnostic Skin Biopsy, IRCCS Fondazione Salvatore Maugeri, Pavia, Italy; ${ }^{6}$ IRCCS Multimedica Institute, Milan, Italy; ${ }^{7}$ Department of Molecular Medicine, University of Pavia, Pavia, Italy and ${ }^{8}$ Cardiovascular Genetics Program, Leon H Charney Division of Cardiology, New York University School of Medicine, New York, NY, USA

*Corresponding authors: G Condorelli, Laboratory of Cardiovascular Reseach, Humanitas Clinical and Research Center, via Manzoni 56, Rozzano (MI) 20089, Italy. Tel: + 3902 82245201; Fax: + 3902 82245290; E-mail: gianluigi.condorelli@ humanitasresearch.it

or SG Priori, Molecular Cardiology, IRCCS Fondazione Savatore Maugeri, via S. Maugeri 10, Pavia (PV) 27100, Italy. Tel: + 390382 592040; Fax: + 390382 592059; E-mail: silvia.priori@fsm.it

${ }^{9}$ These authors contributed equally to this work

${ }^{10}$ Current address: Humanitas Clinical and Research Center, Rozzano (MI), Italy

${ }^{11}$ Current address: Laboratorio de Cardiología Molecular, Instituto de Fisiología, Benemérita Universidad Autónoma de Puebla, Puebla, México Keywords: induced pluripotent stem cells; diseases modeling; cardiomyocytes; CPVT; calcium/calmodulin pathway

Abbreviations: AP, action potential; APD, action potential duration; APD30, action potential duration at $30 \%$ of repolarizarion; APD50, action potential duration at $50 \%$ of repolarization; APD90, action potential duration at $90 \%$ of repolarization; CaMKII, $\mathrm{Ca}^{2+} /$ calmodulin-dependent serine-threonine protein kinase II; CASQ2, calsequestrin 2; CD-15 or SSEA1, stage-specific embryonic antigen 1; CM, cardiomyocyte; CPVT, catecholaminergic polymorphic ventricular tachycardia; DADs, delayed after depolarizations; DAPI, 4',6-diamidino-2-phenylindole; $\mathrm{dCa}^{2+} / \mathrm{d}_{\max }$, rate of intracellular calcium increase; EBs, embryoid bodies; ECG, electrocardiogram; ES, embryonic stem cells; FACS, fluorescence-activated cell sorting; FBS, fetal bovine serum; FH, fetal heart; Fluo-4, 2-\{[3-(2-\{2-[bis(carboxymethyl)amino]-5-(2,7difluoro-6-hydroxy-3-0xo-3H-xanthen-9-yl)phenoxy\}ethoxy)-4-methylphenyl](carboxymethyl)amino\}acetic acid; FGF, fibroblast growth factor; GATA6, GATA-binding factor 6; HEK-293T, human embryonic kidney 293 T cells; HGPRT, hypoxanthine-guanine phosphoribosyltransferase; iPSC, induced pluripotent stem cells; Iso, isoproterenol; KN-92, 2-[N-(4-methoxybenzenesulfonyl)]amino- $N$-(4-chlorocinnamyl)- $N$-methylbenzylamine, phosphate; KN-93, 2-[N-(2-hydroxyethyl)]- $N$-(4methoxybenzenesulfonyl)]amino- $N$-(4-chlorocinnamyl)- $N$-methylbenzylamine; KRT14, epidermal keratin 14 ; MDP, maximum diastolic potential; MEF, mouse embryonic fibroblasts; $\mathrm{MHC} \alpha$, myosin heavy chain, subunit $\alpha ; \mathrm{MHC} \beta$, myosin heavy chain, subunit $\beta$; MSE, mean standard error; NCAM1, neural cell adhesion molecule 1; OCT4, octamer-binding transcription factor 4; PCR, polymerase chain reaction; PECAM1, platelet/endothelial cell adhesion molecule 1; PFA, paraformaldehyde; RyR2, ryanodine receptor, type 2; SOX17, SRY-box containing gene 17; SOX2, SRY (sex determining region Y)-box 2; SSEA4, stage-specific embryonic antigen 4; TA, triggered activity; TRA1-60, tumor rejection antigen 1-60; WT, wild type

Received 02.7.13; revised 25.7.13; accepted 29.7.13; Edited by G Melino
} 
The usefulness of reprogrammed cells in modelling diseases in vitro has recently been tested for several human congenital cardiovascular diseases: iPSCs have been used for the investigation of monogenic disorders (i.e. long QT (prolongation of $\mathrm{Q}-\mathrm{T}$ interval combined with torsades de pointes and manifests as several different forms) syndromes, Brugada syndrome/cardiac conduction disease, catecholaminergic polymorphic ventricular tachycardia and other forms of congenital arrhythmias) and disorders in which cardiac defects are part of a multiorgan phenotype (i.e. Leopard and Timothy syndromes). These reports confirmed that patientspecific iPSCs are able to give rise to differentiated CMs that possess the main functional and morphological aberration typical of the disease in vivo. ${ }^{1,4-13}$ Furthermore, the importance of iPSC in drug discovery applications is useful to identify novel therapeutic molecules and devise patientspecific therapies. $5,6,8$

In our study, we focused on catecholaminergic polymorphic ventricular tachycardia (CPVT), a highly lethal inherited arrhythmogenic disease that may cause syncope and sudden death early in life owing to adrenergically mediated bidirectional ventricular tachycardia. Two genetic forms of the disease have been described: one transmitted as an autosomal dominant trait, caused by mutations in the gene encoding the ryanodine receptor type 2 (RyR2), and another very rare form due to recessive mutations in the cardiacspecific isoform of the calsequestrin gene, CASQ2 (calsequestrin 2). ${ }^{14,15}$ Both genes are involved in regulating $\mathrm{Ca}^{2+}$ handling within the $\mathrm{CM}$ and thus are key in determining excitation-contraction coupling. ${ }^{16,17}$

Although the mortality rate associated with the disease is extremely high (30-35\% by the age of 35 years), therapies are limited. Treatment with $\beta$-adrenergic blockers are usually effective in preventing recurrences of arrhythmias in the majority of patients, but approximately $30 \%$ of patients still experience at least one episode of life-threatening adrenergically induced arrhythmia (leading to syncope or cardiac arrest) despite therapy and will need implantable cardioverter defibrillators. ${ }^{18,19}$

Therefore, development of model systems facilitating screening of new therapeutic molecules for the treatment of CPVT is highly advisable.

Among the putative players in determining the CPVT phenotype, $\mathrm{Ca}^{2+} /$ calmodulin-dependent serine-threonine protein kinase II (CaMKII) has been recently implicated in arrhythmic events elicited by $\beta$-adrenergic activation, and we recently demonstrated that its inhibition is able to prevent ventricular arrhythmogenesis in a mouse model of CPVT. ${ }^{20-22}$

With these considerations in mind, our intent was to create a patient-specific cell-based system that could be used as an in vitro model to facilitate the screening of new therapeutic molecules for the treatment of CPVT. For this purpose, we generated an iPSC-based cardiac model from a patient carrying a heterozygous mutation in the gene encoding RyR2 and with phenotypic manifestations of the disease. In a first instance, we verified that the disease phenotype was recapitulated in the CMs derived from these iPSC. Subsequently, we inhibited the $\mathrm{Ca}^{2+}$-CaMKII pathway, which affects calcium handling, to test whether we could rescue the disease phenotype in human cardiac cells to confirm the clinical relevance of the observation made in myocytes derived from knock-in mice carriers of a heterozygous defect in RyR2 and presenting the clinical phenotype of CPVT.

Our results support the view that iPSC technology is likely to have clinical applicability to predict response to therapy in individual patients.

\section{Results}

Clinical history. In June 2006, the team of our outpatient clinic for inherited arrhythmia at the Maugeri Foundation was contacted for the assessment of a family with a history of juvenile sudden cardiac death. The proband (Figure 1A, subject II-2), a 42-year-old female reported that two of her children died suddenly before age 10 years (Figure $1 \mathrm{~A}$, subjects III-1 and III-2) both in a condition of adrenergic stress. III-1 died at the age of 8 years while riding on a carousel and III-2 died suddenly at the age of 9 years running in a school competition. The mother also reported that III-1 experienced a syncopal spell during physical activity a few months before dying. At that time, the boy was taken to the emergency room, but resting electrocardiogram (ECG) and echocardiogram were unremarkable and he was discharged. The other child of the proband, that is, III-2, died at the age of 9 years with no previous symptoms. At first clinical evaluation, the mother (II-2) reported two previous episodes of loss of consciousness during physical activity (at the age of 41 and 42 years) and reported that in a previous exercise stress test there was documentation of isolated premature ventricular contractions and a ventricular couplet that resulted in the interruption of the test. We recorded her resting ECG (Figure 1B) and echocardiogram, which were unremarkable. However, maximal exercise stress test documented the onset of sustained bidirectional ventricular tachycardia (Figure 1B). CPVT diagnosis was established and $\beta$-blocker therapy was administered. A second exercise stress test after 5 days of therapy with nadolol $(2 \mathrm{mg} / \mathrm{kg})$ showed suppression of arrhythmias after maximally tolerated effort. The patient has remained asymptomatic, with no evidence of arrhythmias as of September 2012. Sequencing of the entire open reading frame of the $R y R 2$ gene identified the c.6933 $\mathrm{G}>\mathrm{C}$ nucleotide transversion in exon 46, leading to the p.Glu2311Asp missense mutation. Unfortunately, no post-mortem samples were available for the deceased children. Genetic testing also identified the same mutation in the asymptomatic two-year-old daughter (III-3), who was promptly treated with oral nadolol $(2 \mathrm{mg} / \mathrm{kg})$. Holter monitoring off therapy showed rare supraventricular and ventricular ectopic beats that disappeared after therapy.

Generation of patient-specific CPVT-iPSC and their characterization. CPVT-iPSCs were generated from primary fibroblasts isolated from a skin biopsy of the proband through lentiviral transduction with OCT4 (octamer-binding transcription factor 4), SOX2 (SRY (sex determining region $Y$ )-box 2), NANOG (homeobox transcription factor) and LIN-28 (zinc-finger CCHC domain-containing protein 1).

Before induction, isolated primary skin cells exhibited the morphology (Figure 1Ca) and antigenic expression pattern of human fibroblasts (Supplementary Figure 1). Several 
A

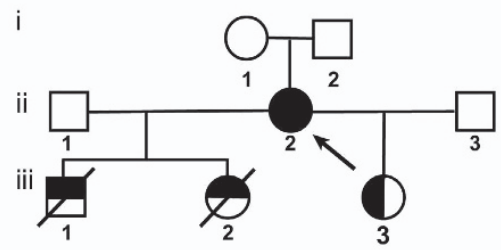

Genetically affected

Clinically and Genetically affected Sudden Cardiac Death

\section{B}

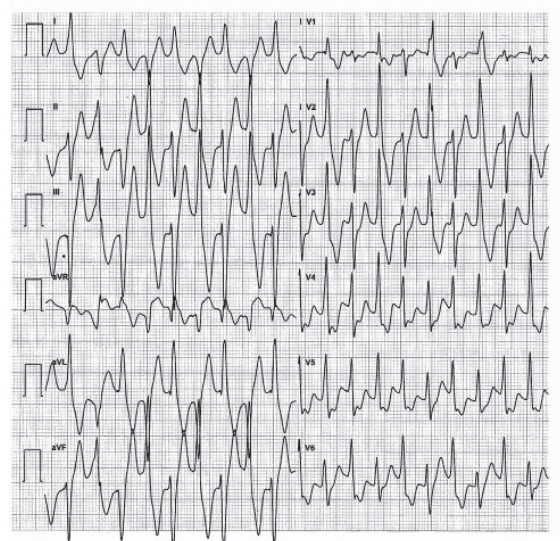

C
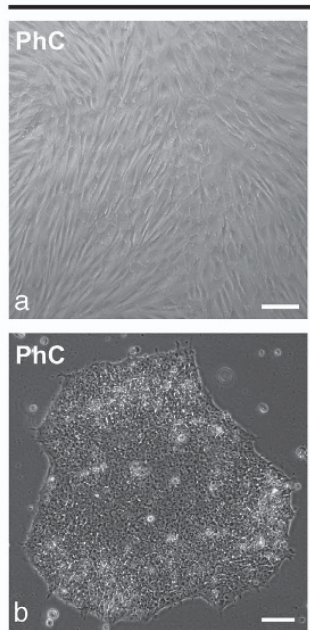

AP

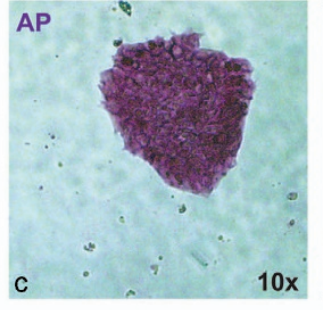

CPVT-iPSC
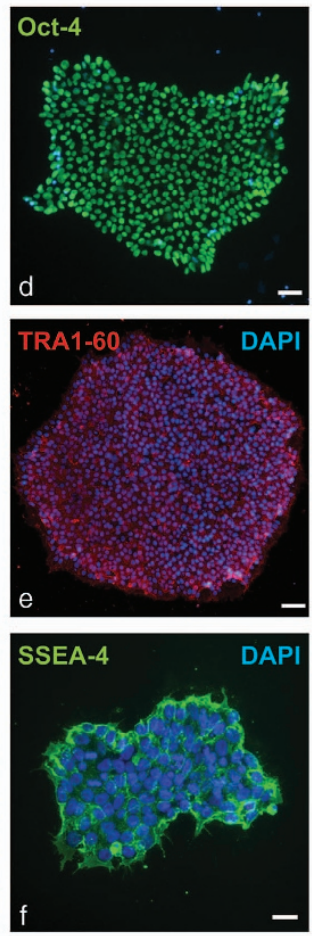

D
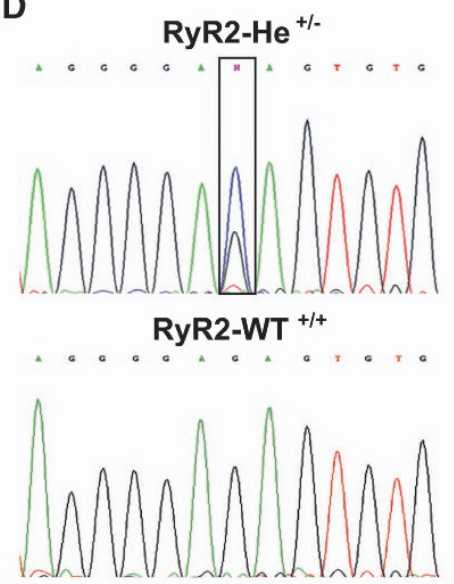

$\mathrm{E}$

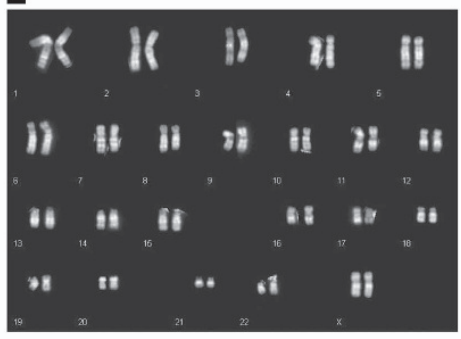

Figure 1 Generation of iPSC from a CPVT patient skin biopsy. (A) Pedigree of the RyR2-He + l- CPVT kindred modeled in this study. Proband (II-2) is indicated by an arrow. Filled symbols indicate clinically and genetically affected subjects. Half-black symbols indicate genetically affected individuals, and upper half-black symbols indicate sudden cardiac death cases. Square = male; circle = female. (B) Example of bidirectional ventricular tachycardia recorded off-therapy in the proband (paper speed $25 \mathrm{~mm} / \mathrm{s}$ ). (C) Representative images of dermal fibroblasts derived from the CPVT patient (a) and of an IPSC colony derived from the patient's fibroblasts (b) showing alkaline phosphatase activity (c) and positivity for the pluripotency markers OCT4 (d), TRA1-60 (e) and SSEA4 (f). Scale bars $=100 \mu \mathrm{m}$. (D) Sequencing analysis confirming that the CPVT-iPSC line (He) carried the specific G-to-C mutation on one allele of the RyR2 gene, whereas control-iPSC (WT) did not show any genetic alteration. (E) iPSC lines maintained a normal karyotype after expansion

patient-specific iPSC clones were generated from them and clones have been selected by their morphological similarity to human ES cells and expanded (Figure 1C). Two iPSC lines were chosen, further characterized and used for differentiating into patient-specific CMs. As a control, iPSCs generated from a healthy subject were used (Supplementary Figure 2). ${ }^{23}$

As a first step, we verified that iPSCs generated were genetically matched to the donor and that those derived from the patient carried the heterozygous p.Glu2311Asp $R y R 2$ gene mutation (RyR2- $\left.\mathrm{He}^{+/-}\right)$, by direct sequencing (Figure 1D). No chromosomal abnormalities were detected by karyotype analysis (Figure 1E).

To establish that reprogramming had occurred correctly and that the selected iPSC clones were pluripotent, we tested whether these lines expressed pluripotency markers by verifying alkaline phosphatase activity ((Figure 1Cc and Supplementary Figure 2C), the expression of 'stemness'associated antigens (tumor rejection antigen 1-60 (TRA1-60) and stage-specific embryonic antigen 4 (SSEA4)) and transcription factors (OCT4, REX1 (RNA exonuclease 1 homolog), DNA (cytosine-5)-methyltransferase $3 \beta$ (DNMT3B)) with different approaches, that is, immunofluorescence staining (Figure $1 \mathrm{C}$ and Supplementary Figure 2), real-time polymerase chain reaction (PCR) (Supplementary Figure $3 \mathrm{~A}$ ) and fluorescence-activated cell sorting (FACS) analysis (Supplementary Figures 3B and C).

Pluripotent cells are by definition capable of differentiating into all cell types of the body. Accordingly, iPSCs are able to spontaneously differentiate into cell types derived from each of the three germ layers when cultured in suspension to form EBs. To test the developmental properties of the selected iPSC lines, we induced differentiation with the EB aggregation method: immunohistochemical analysis (Figure $2 \mathrm{~A}$ and Supplementary Figure 4) and semiquantitative real-time PCR (Figure 2B) revealed that the EBs contained cells expressing markers of the ectodermal (NCAM1 (neural cell adhesion molecule 1), KRT14 (epidermal keratin 14), $\beta$ III-tubulin, nestin), mesodermal ( $\alpha$-smooth muscle actin, desmin, PECAM1 (platelet/endothelial cell adhesion molecule 1) and cardiac genes) and endodermal (GATA6, SOX17 (SRY-box containing gene 17) and $\alpha$-fetoprotein) lineages.

Moreover, control- and CPVT-iPSC injected into immunocompromised mice had the ability to form teratomas containing derivatives of all the three germ layers. This provided more stringent evidence of the pluripotency of these lines (Figure 2C).

Altogether, these data indicate that we have reprogrammed fibroblasts from a patient with CPVT into iPSC. 
A
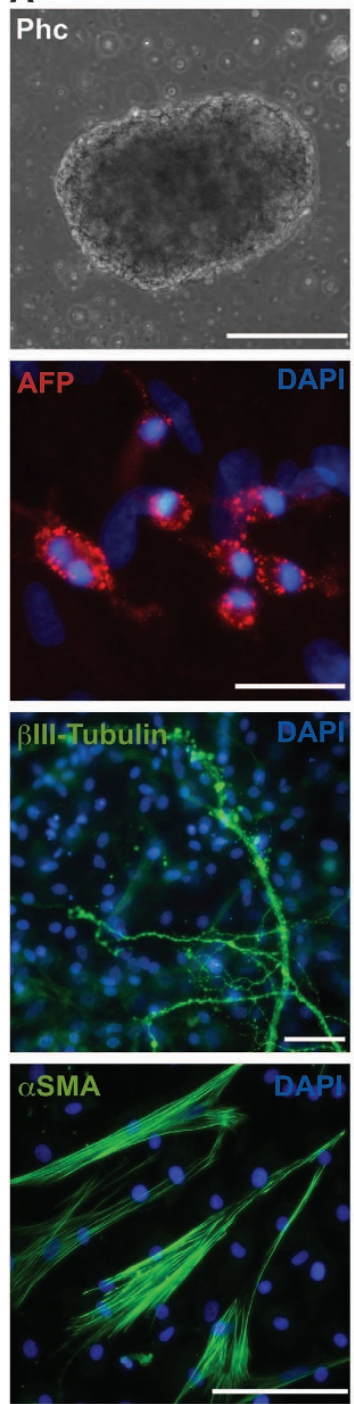

B
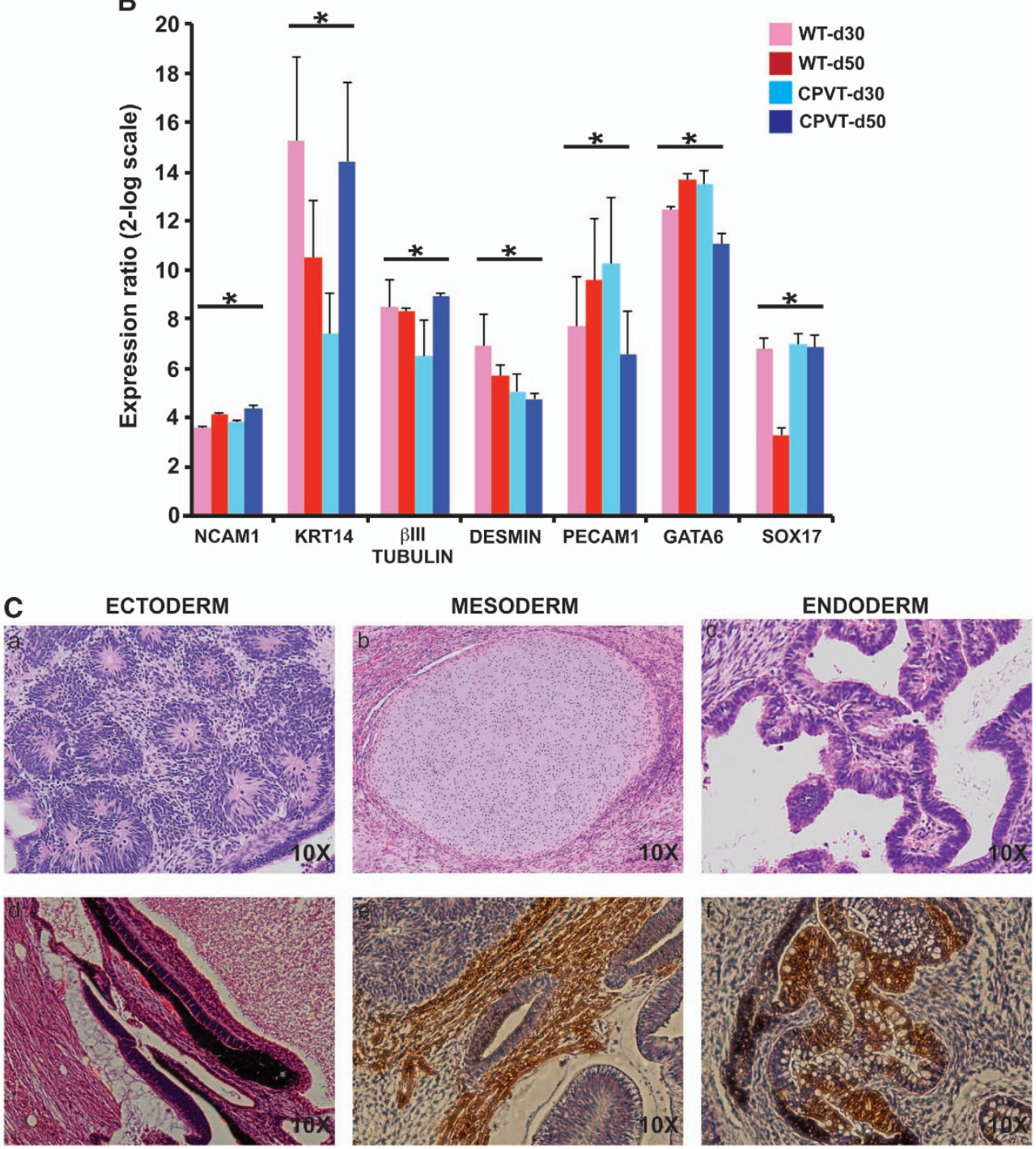

Figure 2 Developmental properties of CPVT-iPSC confirm their pluripotency. (A) Phase-contrast (Phc) image of EBs from CPVT-iPSC at day 6 after formation. Immunostaining of differentiated CPVT-iPSC showing EBs containing cells representative of each of the three embryonic germ layers: endoderm ( $\alpha$-fetoprotein for intestinal cells), ectoderm ( $\beta$ III tubulin for neuronal cells) and mesoderm ( $\alpha$-smooth muscle actin for skeletal muscle, $\alpha$ SMA); nuclei were stained with DAPI. Scale bars $=100 \mu \mathrm{m}$; (B) semiquantitative real-time PCR of differentiated control- (WT) and CPVT-iPSC at days 30 and 50 of differentiation, showing upregulation of expression of markers of the three germ layers: positivity for NCAM1, $\beta$ III-tubulin and KRT14 was indicative of ectodermal cells (neurons or epidermis); the presence of DESMIN and PECAM1 indicated the presence of mesodermal cells; and the transcription factors GATA6 and SOX17 were indicative of endodermal differentiation. Data are presented relative to undifferentiated iPSC and were normalized to HGPRT (hypoxanthine-guanine phosphoribosyltransferase) and GAPDH (glyceraldehyde 3-phosphate dehydrogenase). Values are mean \pm S.D. ${ }^{*} P<0.05$; (C) teratoma formation assay: hematoxylin-eosin staining (a-c) and immunohistochemistry (d-f) of teratomas formed from CPVT-iPSC (representative images from one cell line), showing differentiation of cells injected in vivo into various tissues derived from all the three germ layers: retinal epithelium and neural rosettes derive from ectoderm (d); cartilage and muscle (positivity for $\alpha$-actinin) are mesodermal tissues (e); whereas the presence of respiratory and intestinal (cytokeratin-20 (CK-20) positive) epithelium is indicative of endodermal differentiation (f)

Cardiac differentiation. As a next step, we induced iPSC to differentiate toward the cardiac lineage. Control- and CPVTiPSC lines developed spontaneously contracting areas (Supplementary Movie 1) expressing cardiac-specific channel and structural genes (Figures 3a and b). Importantly, western blot analysis revealed specific expression of RyR2 in iPSC-derived beating explants, either wild-type (WT) or CPVT, at comparable levels (Figures $3 \mathrm{~b}$ and c). Immunostaining analysis confirmed the presence and the distribution of RyR2 in cells isolated from the contracting areas (Figure 3d and Supplementary Movie 2). ${ }^{6,24}$
Characterization of iPSC-derived CMs reveals differentiation of heterogeneous populations of cardiac cells. As a first step in the characterization process, we evaluated the electrical activity of control- and CPVT-iPSC-derived CMs.

The analysis of the main electrical features evidenced that the mean amplitude of the AP was around $20 \mathrm{mV}$ higher in CPVT-CMs than in control-CMs, whereas the action potential duration at 30,50 and $90 \%$ of repolarization $\left(\mathrm{APD}_{30}, \mathrm{APD}_{50}\right.$ and $A P D_{90}$ respectively), the maximum diastolic potential (MDP) as well as the maximal rate of depolarization and 
a

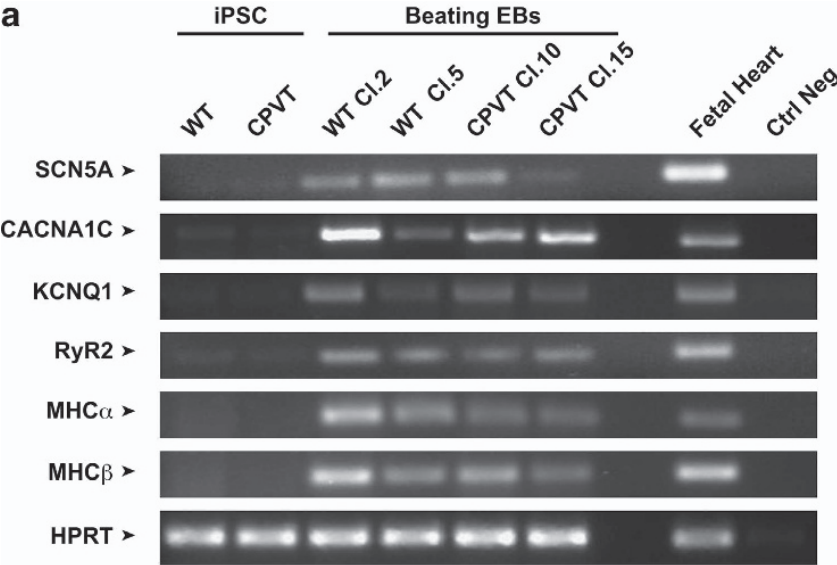

b

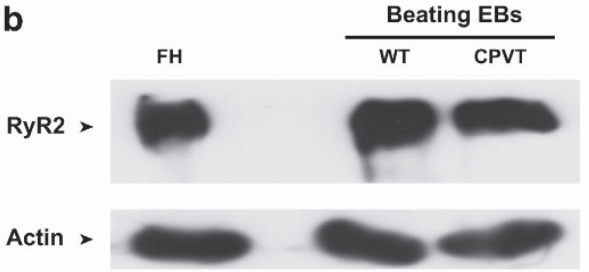

C

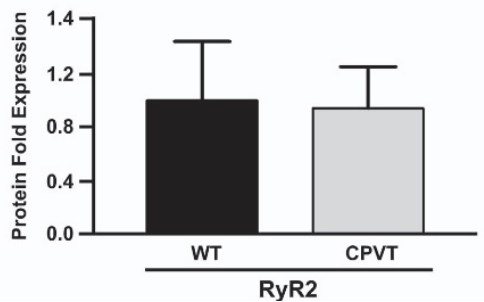

d

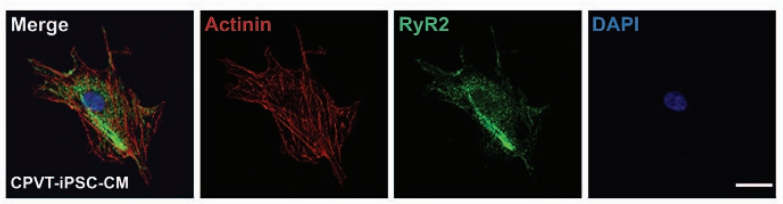

e
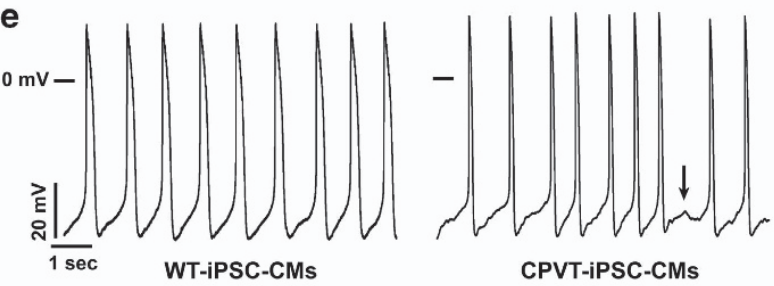

f WT-PSC-CMs
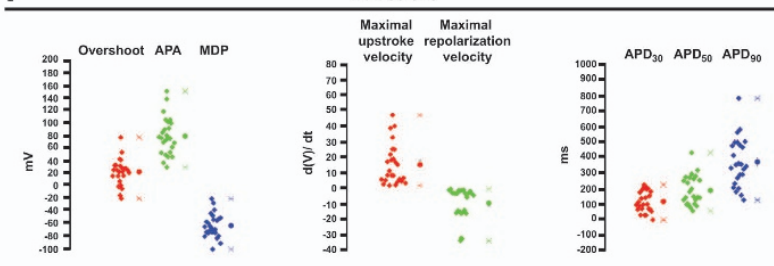

CPVT-PSC-CMs

Maximal
upstroke
velocity
$\begin{gathered}\text { Mapolarization } \\ \text { velocity }\end{gathered}$

Figure 3 iPSC can differentiate into functional CMs. (a) Reverse transcription-polymerase chain reaction (RT-PCR) for the expression of cardiac-specific genes in control(WT) and CPVT-iPSC-derived beating explants (beating EBs); undifferentiated IPSCs and FHs were used as negative and positive controls, respectively. HGPRT: housekeeping gene; CACNA1C: calcium channel, voltage-dependent, $\alpha 1 \mathrm{~A}$ subunit; SCN5A: sodium channel, voltage-gated, type V, alpha subunit; KCNQ1: potassium, voltage-gated channel, KQT-like subfamily, member 1; MHC $\alpha$ : myosin heavy-chain $\alpha ;$ MHC $\beta$ : myosin heavy chain $\beta$. (b) Western blot of WT-and CPVT-IPSC-derived beating explants for RyR2. $\beta$-Actin was used as the loading control, and human FH was used as a positive control. (c) RyR2 expression quantification in WT- and CPVT- beating explants, calculated as densitometry RyR2/ $\beta$-actin ratio (the diagram represents the mean of four independent experiments). (d) Immunostaining of CPVT-iPSC-derived CMs for $\alpha$-actinin and RyR2. Nuclei stained in DAPI. Scale bar $=20 \mu \mathrm{m}$. (e) Representative traces of spontaneous APs recorded in iPSC harvested from the healthy donor (WT-iPSC-CMs, left) and the patient carrying the CPVT mutation (CPVT-iPSC-CMs, right). A DAD is indicated by the arrow. (f) The main AP features measured in both iPSC-derived lines: overshoot, amplitude, MDP, maximal upstroke velocity, maximal repolarization velocity and AP duration at $30 \%, 50 \%$ or $90 \%$ of repolarization (respectively $\mathrm{APD}_{30}, \mathrm{APD}_{50}$ and $\mathrm{APD}_{90}$ ). WT-iPSC-CMs, $n=26$; CPVT-iPSC-CMs, $n=35$. Values are mean \pm MSE

maximal upstroke velocity were similar in both groups (Figure 3f).

Importantly, during the diastolic depolarization phase, CPVT-CMs had delayed afterdepolarizations (DADs), a prominent feature of mature CMs with a CPVT phenotype (Figure 3e).

Furthermore, as already reported in the literature, a more detailed electrical characterization of both control and CPVT cells lines showed that the differentiation process of these cells reflected the heterogeneity seen in the heart. ${ }^{3,11,24}$

In particular, analyzing the cells for a variety of parameters, including the maximal upstroke velocity ( $\left.d V / d t_{\text {max }}\right), A P D_{50}$, $\mathrm{APD}_{90}$ and $\mathrm{AP}$ amplitude, it was possible to cluster two distinct populations of iPSC-derived CMs (iPSC-CMs) in both the cells line: nodal cells (i.e. cells from the AV node), which were distinguishable because of their pronounced phase 4 depolarization preceding the onset of the AP, and working myocardial cells (i.e. atrial and ventricular chamber), which presented the typical plateau phase and, therefore, had the longest AP duration (Supplementary Figure 5). ${ }^{11,24}$

\section{$\beta$-Adrenergic stimulation-induced DADs and triggered} activity in CPVT-CMs. To assess the effect of $\beta$-adrenergic stimulation on CPVT-CMs, we recorded evoked (Figure 4a) and spontaneous (Figure 4b) APs before and after superfusion with the $\beta$-adrenergic agonist isoproterenol (Iso) $(1 \mu \mathrm{M})$. CPVT-CMs presenting spontaneous AP developed DADs already at basal conditions in $43 \%$ of the cases (15 out of 35 ), and $\beta$-adrenergic stimulation significantly increased the frequency of this phenomenon, a hallmark of mature CMs from CPVT patients ( $75 \%$ of the cases, 12 out of 16 ; Figure $4 \mathrm{~b}$, indicated with black arrows). Furthermore, CPVTCMs also developed DADs and triggered activity (TA) when paced at $0.5 \mathrm{~Hz}(12 \%, 2$ out of 16 , Figure $4 \mathrm{a})$, confirming the 

Evoked Action Potentials
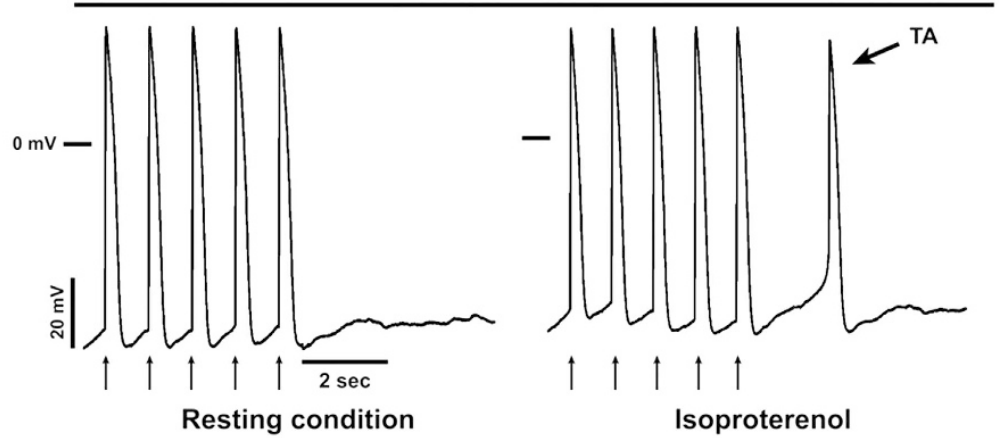

b Spontaneous Action Potentials

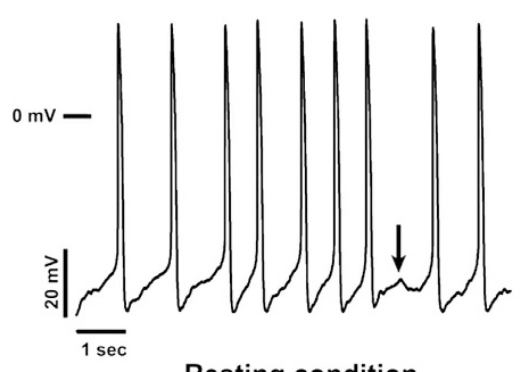

Resting condition

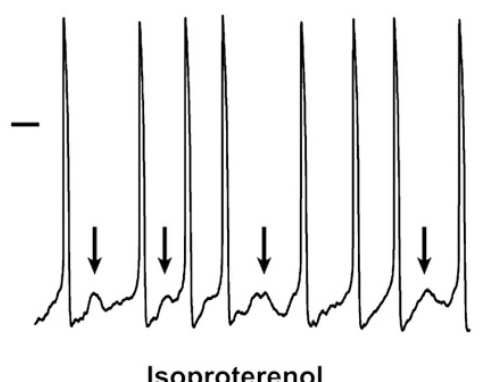

Isoproterenol

c Antiarrhythmic effect of KN-93

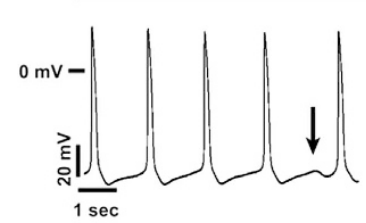

Resting condition

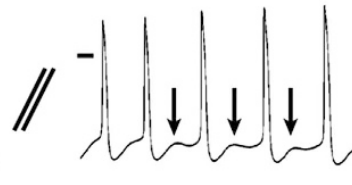

Isoproterenol

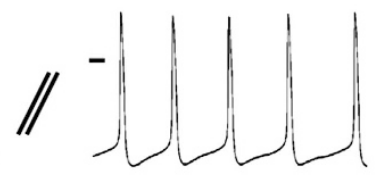

Isoproterenol $+\mathrm{KN}-93$

Figure 4 KN-93 exerts an antiarrhythmic effect on CPVT-iPSC-derived CMs. Representative traces of evoked (a) and spontaneous (b) APs from CPVT-iPSC in the presence of a $\beta$-adrenergic stimulus ( $1 \mu \mathrm{M}$ Iso). DADs are indicated by arrows. (c) Coperfusion with $1 \mu \mathrm{M} \mathrm{KN}-93$, a CaMKIl inhibitor, prevented this arrhythmogenic effect in CPVT-iPSC-derived CMs $(n=7, P<0.05)$

ability of our model system to recapitulate the disease phenotype in vitro.

KN-93 exerts an antiarrhythmic effect on CVPT-CMs. To prove the feasibility of our iPSC-based model for drug discovery and for testing the efficacy of novel therapeutic molecules, we cotreated CPVT-CMs with KN-93 (2-[N(2-hydroxyethyl)]- $N$-(4methoxybenzenesulfonyl)]amino- $N$-(4chlorocinnamyl)- $N$-methylbenzylamine), a CaMKII inhibitor. We recently reported that $\mathrm{KN}-93$ is the most powerful antiarrhythmic agent that prevents ventricular arrhythmias in our RyR2 ${ }^{\mathrm{R} 4496 \mathrm{C}+/-}$ knock-in mouse model of CPVT. $\mathrm{KN}$-93 was also capable of abolishing DADs and TA in isolated $\mathrm{CMs}$ in vitro. ${ }^{21}$

Consistent with the findings obtained in $\mathrm{CMs}$ derived from the CPVT knock-in mice, $1 \mu \mathrm{M}$ KN-93 blunted Iso-induced DADs in iPSC-derived CPVT-CMs $(n=7$, versus Iso, $P<0.05$; Figure 4), whereas the inactive stereoisomer KN-92 (2-[N-(4-methoxybenzenesulfonyl)]amino- $N$-(4-chlorocinnamyl)- $N$-methylbenzylamine, phosphate) $(1 \mu \mathrm{M})$ has no effect on these arrhythmic events ( $n=5, P<0.05$ versus $\mathrm{KN}-93)$.
Importantly, the AP morphology of control-iPSC-derived CMs did not undergo any noticeable changes when exposed to KN-93 (data not shown).

We repeated the same protocol using $3 \mathrm{D}$ beating clusters of control- and CPVT-iPSC-derived CMs, optically assessing intracellular calcium transients after loading with Fluo-4 (2-\{[3(2-\{2-[bis(carboxymethyl)amino]-5-(2,7-difluoro-6-hydroxy3-oxo-3H-xanthen-9-yl)phenoxy\}ethoxy)-4-methylphenyl] (carboxymethyl)amino\}acetic acid). We found that in CPVT-CMs, calcium transients initiated from multiple locations within each beating cluster ( $n=8$; Figure $5 b$ and Supplementary Movie 3) and that the impulses collided during the propagation, generating a non-homogeneous spreading of calcium transients. This condition was exacerbated by $\beta$-adrenergic stimulation during superfusion of the preparation with Iso. In this setting, the number of areas generating spontaneous calcium transients was increased. When we superfused the CPVT multicellular preparations, we observed that $\mathrm{KN}-93$ stabilized calcium activation, reverting to the typical pattern observed in normal tissue, characterized by a single initiation site located on the periphery of the preparation where the 
a
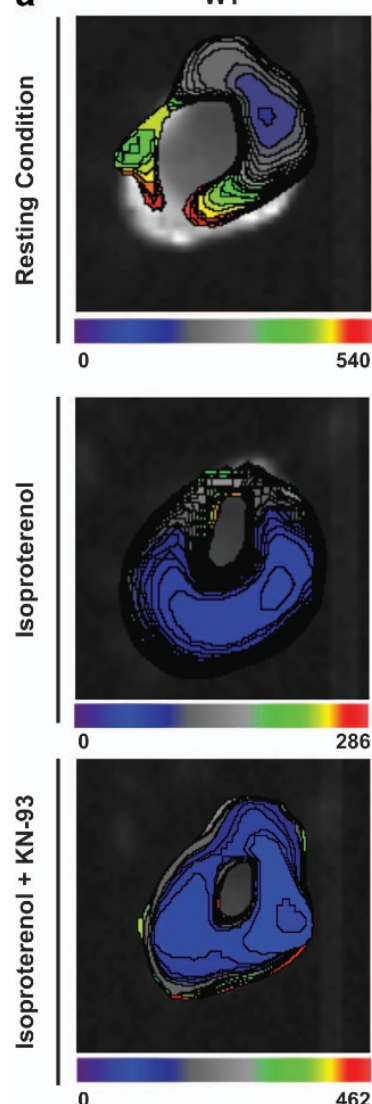
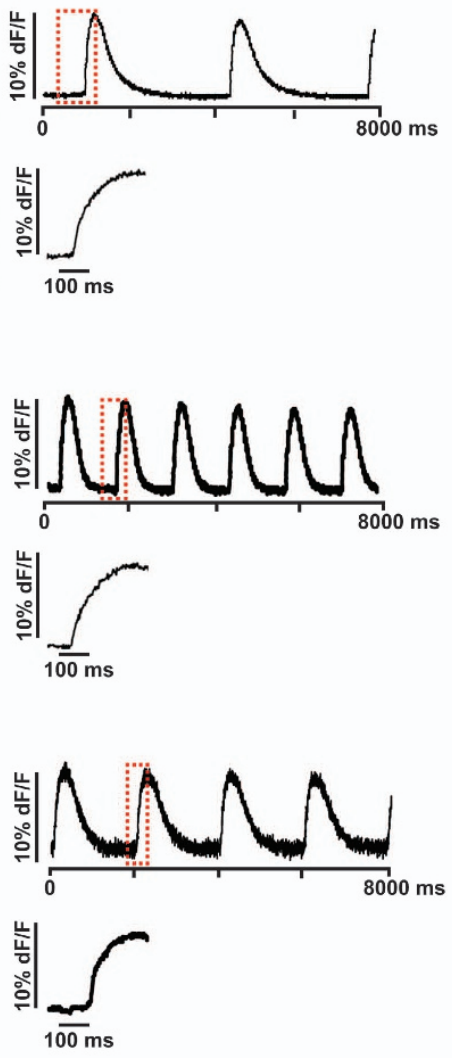

b

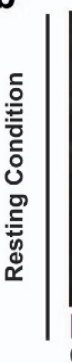

CPVT
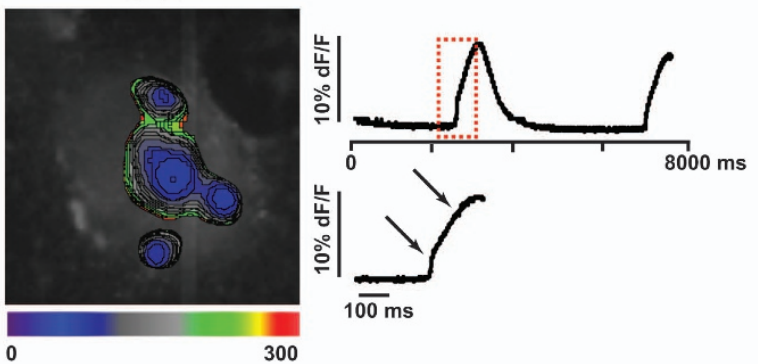
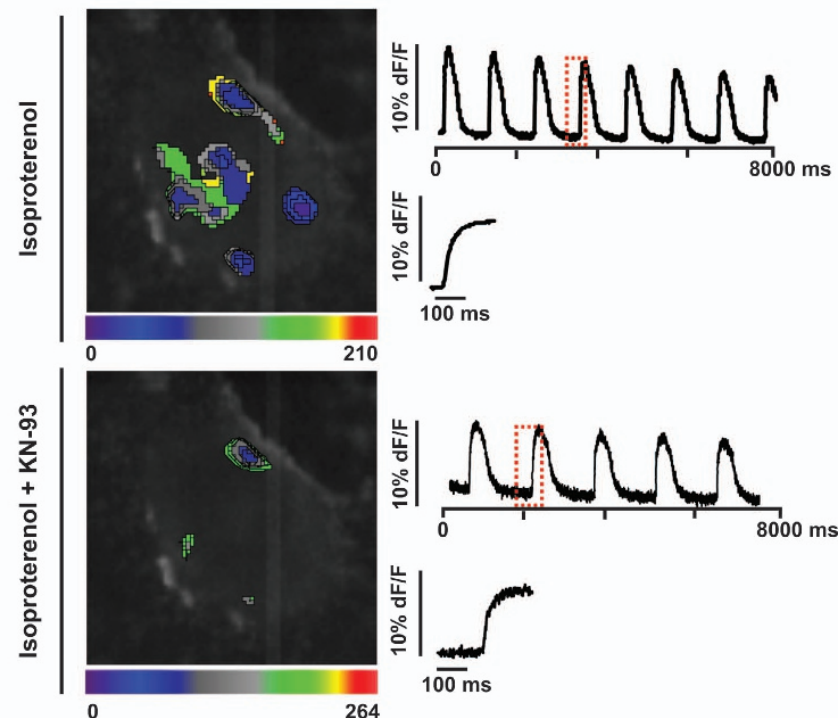

Figure 5 Calcium transient detection in iPSC-derived 3D-beating clusters. (a) Calcium transient activation time map and representative traces of WT cluster recorded in basal conditions (top), in the presence of a $\beta$-adrenergic stimulus $(1 \mu \mathrm{M} \mathrm{Iso})$ (middle) and in coperfusion with $1 \mu \mathrm{M} \mathrm{KN}-93$ (bottom) $(n=6)$. Dashed red lines indicate the zoomed-in regions of the calcium upstroke represented below. (b) Same as (a) for CPVT clusters $(n=8)$. All traces are scaled to control value as normalized dF/F $10 \%$. Rainbow line indicates the isochrones of calcium impulse initiation and propagation

source-to-sink load was favorable. ${ }^{25}$ As expected, control beating clusters had a single region of calcium impulse initiation under basal conditions and during Iso administration $(n=6$; Figure $5 \mathrm{a}$ ). Moreover, in $75 \%$ of the experiments (six out of eight), the upstroke of the $\mathrm{Ca}^{2+}$ transient in CPVT clusters in the presence of Iso had a double slope before reaching the peak (Figure $5 \mathrm{~b}$, middle panel). To note, $\mathrm{KN}-93$ recovered this abnormal feature of the calcium upstroke. This may explain why the rate of intracellular calcium increase $\left(\mathrm{dCa}^{2+} / \mathrm{d} t\right)$ after the addition of the CaMKII inhibitor slightly decreased (Figure 6c, versus Iso, not statistically significant), whereas the time to reach the peak was significantly reduced $(P<0.05$, versus Iso; Figure 6b).

\section{Discussion}

A little more than a decade ago, mutations in the cardiac ryanodine receptor gene $(R y R 2)$ were first associated with CPVT, a life-threatening inherited arrhythmogenic disorder. ${ }^{15}$ Since then, much has been learnt about the pathogenesis of this disease: experimental findings from lipid bilayers as well as knock-in and knockout mouse models suggested that the mechanism underlying the onset of arrhythmia in CPVT patients strictly relies on defective $\mathrm{Ca}^{2+}$ mobilization within the $\mathrm{CM}$ during excitation-contraction coupling. Diastolic $\mathrm{Ca}^{2+}$ leak from the sarcoplasmic reticulum is believed to be the major player for the development of DADs, typical markers of electrical instability in CPVT-CMs. DADs are elicited by intracellular calcium load, which activates the membrane $\mathrm{Na}^{+} / \mathrm{Ca}^{2+}$ exchanger in an electrogenic mode derived by the exchange of one $\mathrm{Ca}^{2+}$ for three $\mathrm{Na}^{+}$, leading to diastolic membrane depolarizations that may reach the activation threshold for inward sodium current and generate triggered beats that may eventually lead to sustained arrhythmias. ${ }^{26,27}$

The development of novel therapeutic approaches has been limited and the use of implantable defibrillators remains the therapy of choice for patients unresponsive to the therapeutic options. Furthermore, the only disease models of CPVT are the knock-in mice that have been used by us, and others, to test new drugs. ${ }^{21}$ However, the results obtained in myocytes from mice leaves investigators with the uncertainty of whether the antiarrhythmic effect seen is replicated in humans.

Clearly, the inability to study the disease and test new treatments in human diseased CMs represents a major limitation. Furthermore, accessibility to human cardiac tissue is limited to heart surgery or to post mortems.

The advent of human iPSC technology may solve these issues and revolutionize the investigation of pathological molecular events driving human diseases: these cells offer an 

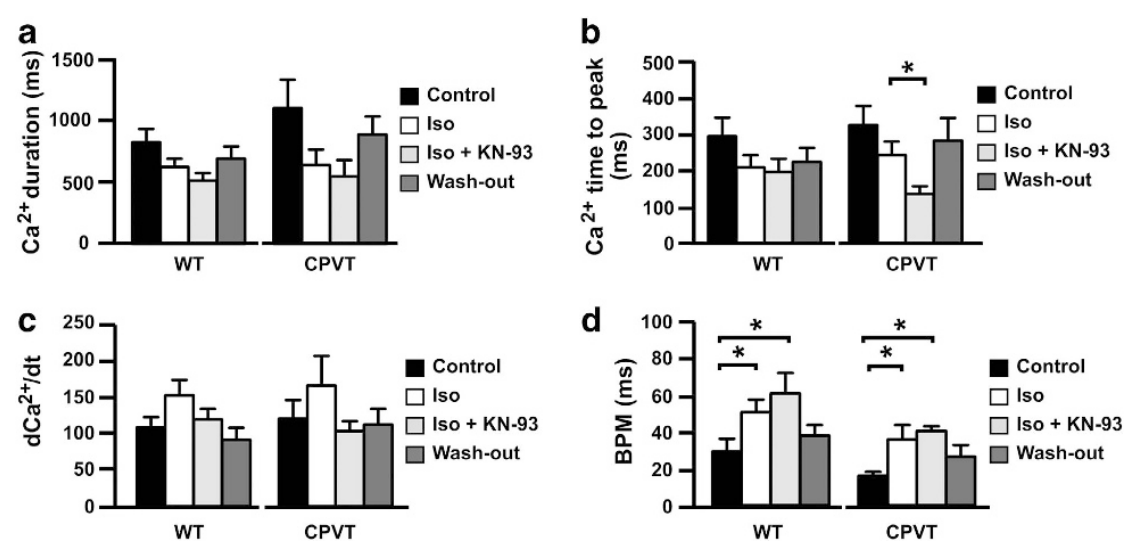

Figure 6 Calcium transient measurements. Schematic representation of the calcium transient measurements by optical mapping fluorescence showing calcium duration (a), calcium time to peak (b), $\mathrm{dCa}^{2+} / \mathrm{d} t$ (percentage $\mathrm{Ca}^{2+}$ potential amplitude per s) (c) and beat per minutes (b.p.m.) (d) ( $\left.{ }^{\star} P<0.05\right)$

inexhaustible and scalable source of differentiated patientspecific CMs that carry the same genome as the subject they are derived from, and in which mechanisms of the disease and the response to drugs may be investigated. ${ }^{28}$

This study confirms the potential for human iPSC to model inherited arrhythmogenic syndromes by establishing a patient-specific model of CPVT in which to test therapeutic interventions that have been successful in our murine model of CPVT.

We performed a systematic study of the electrical properties of iPSC-derived CMs from WT and CPVT genotypes using the whole-cell patch clamp technique on spontaneously beating cells at 90 days after the formation of embryoid bodies (EBs). We did not find great heterogeneity in the electrical parameters between the two different groups of cells, but it was possible to highlight a pronounced withingroup variability. Furthermore, although the distinction between atrial- and ventricular-like myocytes was not so defined, because of the existence of cells with an intermediate phenotype, we were able to cluster the cells into 'working-like' and 'nodal-like' CMs. ${ }^{6,11,24}$ However, the impressive heterogeneity in the electrical parameters is a striking feature of the published electrophysiology data on iPSC-derived myocytes. This could be because of the different stage of maturation of the cells and/or to the sophisticated architecture of the electrical structure of the heart reproduced in vitro. ${ }^{3}$

Importantly, whole-cell patch clamp and microelectrode recordings revealed that CPVT-iPSC-derived CMs exhibited the key features of catecholaminergic-mediated arrhythmogenesis. Hence, clear DADs were observed in most of the CPVT-iPSC-CMs studied. This became even more prominent after activation of the $\beta$-adrenergic signaling pathway and gave rise to TA beats.

We also performed impulse-initiation optical mapping in order to determine the electrophysiological phenotypic defects in the context of the spontaneous beating cluster and thus to seek the main electrical disturbance of CPVTinduced arrhythmia. We have used this technique for the first time to build a spatiotemporal high-resolution map for studying the characteristics of intracellular calcium propagation in the context of the whole CPVT cluster. We detected that calcium transients were initiated within a delay of a few milliseconds from multiple regions within the cluster; this resulted in a double rise in the intracellular calcium transients, leaving the reuptake not affected. This is in contrast with recent observations by Novak and co-workers, ${ }^{13}$ where a prominent diastolic calcium rise was demonstrated as an indication of DADs. This discrepancy may be due to the diverse technical approaches employed to optically assess calcium transients (ratiometric versus non-ratiometric dyes). On this point, it is worth noting that both types of dyes possess high affinity for $\mathrm{Ca}^{2+}$, and this can artificially prolong the calcium transient and confound interpretation in CMs and tissues. ${ }^{29}$ On the other hand, the fast chelation of Fluo-4 permits a better evaluation of the calcium upstroke. A different degree of differentiation of the beating cluster may also be a source of variability among generated data.

Our results offer a 'side' view of CPVT, in which the occurrence of calcium transient initiation at multiple foci constitutes the arrhythmogenic substrate by underlying altered gating kinetics of RyR2 (which may explain the double upstroke).

Furthermore, in agreement with data previously obtained in the RyR2 ${ }^{\mathrm{R} 4496 \mathrm{C}+1-}$ CPVT mouse model, ${ }^{21}$ we demonstrate that CaMKII inhibition prevents $\beta$-adrenergically induced arrhythmogenesis also in patient-specific CMs. Thus, this technique opens up the possibility of testing the response to therapy of individual patients within the clinic. This transition from bench to bedside is most exciting. However, the technology needed to generate iPSC-derived CMs is still expensive and time consuming. Nonetheless, we anticipate the advent of novel technology that will reduce the 'biopsy-tohuman-CMs' time.

A few tests of substances as putative therapeutic agents on iPSC-based CPVT models have already been reported. ${ }^{6,10}$ For example, flecainide has been recently proposed as an antiarrhythmic drug in mice and human. However, there are still uncertainties on the mechanism that drives its antiarrhythmic activity. Although some authors believe that flecainide acts by inhibiting RyR2's open state, ${ }^{30,31}$ we supported an alternative hypothesis and demonstrated that the sodium channel blockers of the drug is preventing DADs to activate $/_{\mathrm{Na}}$ and generates triggered automaticity. ${ }^{32}$ This hypothesis was recently supported by Sikkel et al. ${ }^{33}$ Another potential 
therapeutic agent for CPVT is dantrolene, a unique and very effective therapeutic option for malignant hyperthermia: this substance has been shown to act by stabilizing interdomain interaction of RyR2 and decreasing loss of $\mathrm{Ca}^{2+}$ from sarcoplasmic reticulum. ${ }^{6,34,35}$ In the present report, we propose inhibition of CaMKII as a potential therapeutic option for treating arrhythmias in CPVT. CaMKII is activated by multiple pathways and, within the $\mathrm{CM}$, primarily acts by phosphorylating the main components of the calcium handling machinery and, as such, has a clear relevance in the pathophysiology of CPVT. Inhibition of this pathway has been shown to be potentially advantageous compared with $\beta$-blockers, the conventional therapy for CPVT patients; however, the use of CaMKII inhibitors in the clinical setting is still limited by the lack of molecules with target- and tissuespecificity. ${ }^{36}$ The development of a human CPVT model system and the demonstration of its ability to specifically respond to $\mathrm{KN}-93$ (no activity of the inactive analog $\mathrm{KN}-92$ was detected) is instrumental to future investigations on identifying specific targets and devising effective strategies for the use of CaMKII inhibition in the clinical setting.

In conclusion, our work contributes to the implementation of the available CPVT mutant models, which is mandatory for establishing a direct relationship between specific mutations and the observed functional effects, as well as determining potential side effects and is fundamental for validating such findings in the perspective of customized patient treatment.

\section{Materials and Methods}

Cell culture. Dermal fibroblasts were obtained by enzymatic digestion from 3 to $4 \mathrm{~mm}$ skin biopsies of a patient diagnosed with CPVT after written informed consent. Isolated fibroblasts were cultured in DMEM-low glucose/F12 (1:3) supplemented with $10 \%$ fetal bovine serum (FBS), glutamine, $0.1 \mathrm{mM}$ nonessential amino acids and antibiotics.

Mouse embryonic fibroblasts (MEFs) were isolated from E12.5-13.5 embryos, following a standard protocol. ${ }^{37}$ Inactivated MEFs were prepared from cells at passage 3 by treatment with mitomycin $\mathrm{C}(10 \mu \mathrm{g} / \mathrm{ml})$ for $3 \mathrm{~h}$ at $37^{\circ} \mathrm{C}$.

After derivation, iPSCs were initially grown on a MEF feeder layer in human embryonic stem cell (ES) medium, that is, knockout DMEM supplemented with $20 \%$ knockout serum replacement, $2 \mathrm{mM}$ glutamax, $0.1 \mathrm{mM}$ non-essential amino acids, $1 \%$ B27 supplement without vitamin A, $1 \%$ N2 supplement, $0.1 \mathrm{mM}$ $\beta$-mercaptoethanol, $50 \mu \mathrm{g} / \mathrm{ml}$ penicillin, $50 \mu \mathrm{g} / \mathrm{ml}$ streptomycin (all from Invitrogen, Life Technologies, Carlsbad, CA, USA), and $20 \mathrm{ng} / \mathrm{ml}$ human basic- fibroblast growth factor FGF (Miltenyi Biotec, Bergisch Gladbach, Germany). At passage 2-3, iPSC lines were adapted to grow on Matrigel (human ES-qualified Matrix from BD Biosciences, Franklin Lakes, NJ, USA) in mTESR1 medium (Stem Cell Technologies, Vancouver, BC, Canada) as described. ${ }^{23}$

Human iPSC generation and characterization. Reprogramming was induced by lentiviral infection, as described. ${ }^{38,39}$ In brief, lentiviral particles were produced in human embryonic kidney 293T cells (HEK-293T) cells by independent transfections of the four 'pluripotency' genes Oct4, Sox2, Nanog and Lin28 (Addgene plasmids 16 579, 16577, 16578 and 16580 from Thomson Laboratory, University of Wisconsin, Madison, WI, USA) using the calcium phosphate method. ${ }^{40}$ Viral supernatants were collected at $30 \mathrm{~h}$ and used fresh for the infection.

Low-passage fibroblasts were seeded at $8 \times 10^{5}$ cells per $100 \mathrm{~mm}$ dish on the day before the infection. The cells were then infected two times using an equal amount of lentiviral particles for each gene in the presence of $4 \mu \mathrm{g} / \mathrm{ml}$ polybrene. Six days later, infected fibroblasts were seeded onto MEF feeders at a low density $\left(5 \times 10^{4}\right.$ cells per $100 \mathrm{~mm}$ dish). The next day, the medium was replaced with regular human ES cell culture medium supplemented with basic FGF ${ }^{38}$ Valproic acid $(0.5 \mathrm{mM})$ was applied for 10 days $^{41}$ to improve the efficiency of the reprogramming process. iPSC colonies became evident around days $21-25$ after infection and were mechanically isolated based on their ES-like morphology. Isolated clones were expanded and their pluripotency characterized through the evaluation of 'stemness' marker expression and the analysis of their developmental competence in vitro (EBs assay) and in vivo (teratoma formation assay). ${ }^{3}$

Two clones for each subject were used for the experiments.

Immunohistological analysis and alkaline phosphatase activity. Cells were fixed in 4\% paraformaldehyde (PFA) for 20 min and permeabilized with $0.2 \%$ Triton for $10 \mathrm{~min}$. Blocking of unspecific sites was achieved by incubation with $10 \%$ donkey or goat serum (Sigma-Aldrich, St. Louis, MO, USA) for $1 \mathrm{~h}$ at room temperature. Cells were stained with several primary antibodies, specific for either 'stemness' or differentiation markers: human fibroblast surface protein (Clone 1B10, mouse monoclonal, 1:100; Sigma-Aldrich), human Oct4 (mouse monoclonal, 1:500; Millipore, Billerica, MA, USA), human TRA1-60 (mouse monoclonal, 1:100; Stem Cell Technologies), human SSEA-4 (mouse monoclonal, 1:100; Stem Cell Technologies), human $\beta$ Ill-tubulin (mouse monoclonal, 1: 100; Promega, Madison, WI, USA), human nestin (mouse monoclonal, 1:100; Millipore), human smooth muscle actin (mouse monoclonal, 1:20; Dako, Glostrup, Denmark), human $\alpha$-1-fetoprotein (rabbit polyclonal, 1:100; Dako), human $\alpha$-sarcomeric actin (rabbit polyclonal, 1:400; Abcam, Boston, MA, USA), $\alpha$-actinin (mouse monoclonal, 1:500; Sigma-Aldrich) and ryanodine receptor 2 (rabbit polyclonal, 1:100; Alomone labs, Jerusalem, Israel). Alexa-Fluor-488and -555-conjugated secondary antibodies were used for specific detection, whereas nuclei were stained with 4',6-diamidino-2-phenylindole (DAPI). Coverslips were mounted using Vectashield mounting medium (Vector Laboratories, Burlingame, CA, USA). Confocal microscopy was performed with a Leica TCS-SP2 digital scanning confocal microscope equipped with a HCX PL APO $\times 40$ /numerical aperture $=1.25$ oil immersion objective. The pinhole diameter was kept at Airy 1. Images were exported to Adobe Photoshop (Adobe Systems, Mountain View, CA, USA) and created with Adobe illustrator (Adobe Systems).

Alkaline phosphatase activity of IPSC lines was determined using the Alkaline Phosphatase Detection kit (Millipore), after cell fixation in 4\% PFA, according to the manufacturer's instructions. Lines were considered positive when alkaline phosphatase activity was detected in more than $95 \%$ of iPSC lines (two clones each condition were analyzed).

RNA extraction and RT-PCR. Total RNA was isolated using Trizol (Invitrogen), treated with amplification grade DNAse I (Invitrogen) and reverse transcribed to cDNA (Superscript III First-Strand Synthesis System; Invitrogen). Real-time PCR was carried out on an ABI7900HT (Applied Biosystems, Life Technologies, Carlsbad, CA, USA) using either the Taqman Gene Expression Assay or the SybrGreen PCR Master Mix (Applied Biosystems), and data were analyzed with REST (Relative Expression Software Tool) software (http://www.gene-quantification.de/rest.html). ${ }^{42}$

Expression of cardiac-specific genes in cDNA from beating explants was assessed with regular RT-PCR using specific primers. A complete list of the primers used in these experiments is provided in Supplementary Table 1.

Flow cytometry analysis. Dermal fibroblasts and iPSCs were harvested and dissociated into single cells using Trypsin and Tryple Express (Invitrogen), respectively. Surface markers were assessed on fresh cell samples. Anti-CD13APC, anti-CD15-PE, anti-SSEA4-FITC and anti-TRA1-60-PE were from BD Pharmingen (San Diego, CA, USA). Analyzes were carried out on a FACS Canto flow cytometer (Beckton Dickinson, Franklin Lakes, NJ, USA). Data were analyzed with DIVA software (Beckton Dickinson).

Western blot analysis. Whole-cell lysates were obtained from control (WT) and CPVT iPSC-derived beating explants and analyses preformed using $25 \mu \mathrm{g}$ of proteins following standard procedures. Proteins from human fetal heart $(\mathrm{FH})$ were used as positive control. Monoclonal anti-RyR2 (1:1000; Thermo Fisher, Waltham, MA, USA) and polyclonal anti- $\beta$ Actin (1:2000; Santa Cruz Biotechnologies, Dallas, TX, USA) antibodies were used for detection.

Quantification of RyR2 expression levels was determined using Fiji software (Open Source image processing package available at the website: http://fiji.sc/Fiji). ${ }^{36}$

Genomic sequencing and karyotyping. Genomic DNA was isolated from control- and CPVT-derived iPSC lines (two clones each) by DNeasy Blood 
and tissue kit (Qiagen, Venlo, Limburg, Netherlands). Purified DNA was amplified with Bid Dye Terminator v.1.1 Sequencing RR-100 (Applied Biosystem) with specific primers and analyzed with a 3130xl Genetic Analyzer (Applied Biosystem and Hitachi, Chiyoda, Tokyo, Japan). Chromosomal G-banding analysis was performed by the University of Milan-Bicocca Cytogenetics Laboratory (Milan, Italy), using standard procedures.

Spontaneous differentiation and cardiac induction. Control and CPVT iPSCs were differentiated by aggregation into EBs: iPSC colonies were detached using $1 \mathrm{mg} / \mathrm{ml}$ dispase (Roche, Basel, Switzerland) and plated onto ultra-low-attachment plates (Corning, Incorporated, Corning, NY, USA) in EB differentiation medium, that is, DMEMF12 medium supplemented with $20 \%$ FBS, $0.1 \mathrm{mM}$ non-essential amino acids, glutamine and antibiotics. After 7 days, EBs were plated onto gelatin-coated dishes for further differentiation.

For cardiac lineage induction, ascorbic acid (50 $\mu \mathrm{g} / \mathrm{ml})$ was added to the medium. Spontaneously contracting areas, which appeared 12-30 days after EB plating, were manually microdissected and plated onto fibronectin-coated plates for further differentiation for an additional 45-90 days. Explants were maintained in EB differentiation medium supplemented with FBS at only $2 \%$. For single-cell analyses (electrophysiological and immunofluorescence analyses), cells were dissociated as described previously ${ }^{9}$ and plated onto fibronectin-coated plastic or glass 2-well chamber slides (Nunc, Nalge Nunc International, Penfield, NY, USA).

Teratoma assay. iPSC lines were harvested by dispase treatment, resuspended in X-VIVO medium (Lonza, Basel, Switzerland), and injected subcutaneously into immunodeficient mice (NOD-SCID or $\mathrm{Rag}^{-1-}$ (mice homozygous for the scid mutation (severe-combined immunodeficiency) are severely deficient in functional $B$ and $T$ lymphocytes)). Teratomas formed 9-15 weeks after injection were collected and processed according to standard procedures for paraffin embedding and hematoxylin-eosin and immunohistochemical staining.

Recording of APs. Cells were seeded on poly-lysine-like-covered slides (Lab-Tek II, Nunc) and kept in differentiation medium for about 2 months. APs from spontaneously contracting iPSC-CMs were recorded using the patchclamp technique in the whole-cell configuration with a MultiClamp $700 \mathrm{~B}$ amplifier (Axon Instruments, Sunnyvale, CA, USA). The experiments were performed at $37^{\circ} \mathrm{C}$ under continuous perfusion of extracellular solution containing (in $\mathrm{mM}$ ): $140 \mathrm{NaCl}, 4 \mathrm{KCl}, 2 \mathrm{CaCl}_{2}, 1 \mathrm{MgCl}_{2}, 10 \mathrm{HEPES}$ and 5 glucose ( $\mathrm{pH}$ adjusted to 7.40 with $\mathrm{NaOH}$ ). Patch-clamp pipettes, formed from borosilicate glass with a P-97 horizontal puller (Sutter Instruments, Novato, CA, USA), and had a resistance of 2-3M 2 when filled with an intracellular solution containing (in $\mathrm{mM}$ ): $20 \mathrm{KCl}, 120 \mathrm{~K}$-aspartate, $1 \mathrm{MgCl}_{2}, 4 \mathrm{Na}_{2}$-ATP, 0.1 GTP, 10 glucose and 10 HEPES (pH adjusted to 7.20 with $\mathrm{KOH}$ ). Some experiments were carried out with intracellular electrophysiology recordings. In this case, spontaneously beating EBs were impaled using sharp glass microelectrodes with resistances $\geq 10 \mathrm{M} \Omega$. Electrode capacitance was nulled and the recordings were made using the previously described MultiClamp $700 \mathrm{~B}$ amplifier in gap-free mode. Solutions containing $1 \mu \mathrm{M}$ Iso, $1 \mu \mathrm{M} \mathrm{KN}-93$ or $\mathrm{KN}$-92 were prepared fresh before the experiments and applied using a gravitational flow system for 2-3 min before data collection. All signals were acquired at $10 \mathrm{KHz}$, digitized (Digidata 1332A; Axon Instruments) and analysed with pCLAMP 9.2 software (Axon Instruments).

Definition of delayed APs and TA. We defined DADs as low-amplitude depolarizations following completion of repolarization, and have an amplitude $\geq 5 \%$ of the preceding AP. TA was defined as an AP developing from a DAD rather than from an external stimulus.

Fast optical mapping of intracellular calcium transient. Intracellular calcium transient characteristics were measured as described previously. ${ }^{43}$ Briefly, ectopic clusters from CPVT and WT were excised and recultured onto $22 \mathrm{~mm}$ glass coverslip. After $48-96 \mathrm{~h}$, the coverslips were immersed in a $1 \mathrm{ml}$ solution containing culture medium plus $2.5 \mu \mathrm{mol} / /$ of Fluo-4 AM (Invitrogen, Life Technologies) and incubated for $20 \mathrm{~min}$ at $37^{\circ} \mathrm{C}$. Afterwards, the coverslips were mounted onto a custom-made microscope chamber and perfused with Tyrode solution at $37^{\circ} \mathrm{C}$ containing (in $\mathrm{mM}$ ): $140 \mathrm{NaCl}, 4 \mathrm{KCl}, 2 \mathrm{CaCl}_{2}, 1 \mathrm{MgCl}_{2}, 10 \mathrm{HEPES}$ and 5 glucose (pH adjusted to 7.40 with $\mathrm{NaOH}$ ). Optical recording of intracellular calcium transient were assessed using a CMOS fast resolution camera (Ultima L;
Scimedia, Costa Mesa, CA, USA) mounted on an inverted microscope (Nikon Ti/U from Nikon Instruments, Chiyoda, Tokyo, Japan) and acquired for $8 \mathrm{~s}$ at $0.5 \mathrm{KHz}$ at $\times 10$ magnification. To reduce the light exposure, the synchronization of the light shutter and the acquisition was achieved using Digidata 1440A (Molecular Devices, Sunnyvale, CA, USA; Crisel IT) by programming a dedicate protocol of acquisition. Recordings were analysed using BV-Analysis v.1208 (Scimedia).

Statistical analysis. Data are represented as mean \pm MSE (or mean \pm S.D. where indicated). The significance of differences between two groups was evaluated with unpaired Student's $t$-test. $P<0.05$ was considered statistically significant. Single asterisk indicates $P<0.05$, whereas double asterisks indicate $P<0.01$.

\section{Conflict of Interest}

The authors declare no conflict of interest.

Acknowledgements. We gratefully acknowledge Professor James Thomson (through Addgene) for providing the lentiviral vectors for the reprogramming experiments. We also thank Dr. Paolo Vezzoni for his assistance in the teratoma assay experiments, Professor Dalpra' for the karyotype analyses and Dr. Patrizia Vaghi ('Centro Grandi Strumenti' of the University of Pavia) for technical assistance provided for the confocal microscopy experiments. We are particularly grateful to the human subjects that agreed to participate in this study. This work was founded by the 'Superpig' Program co-financed by the Lombardy Region through the 'Fund for Promoting Institutional Agreements' (Institutional Agreements no. 14388A), the PNR-CNR Aging Program 2012-2014 and an 'Advanced' ERC grant (Cardioepigen) to GC; by a Young Researcher Project, Italian Ministry of Health Grant No.GR-20091530528 (to MM); by Telethon Grants Nos. GGP11141 and GGP06007 (to SGP); by a Fondation Leducq Award to the Alliance for Calmodulin Kinase Signaling in Heart Disease (08CVD01) (to SGP) by the PRIN project No. 2010BWY8E9 (to SGP); and by a FondazioneVeronesi Award on Inherited Arrhythmogenic Diseases (to SGP).

\section{Ethical Statement}

The study has been conducted in a safe and ethical manner after the approval of the Institutional IRB. All the subjects involved in the study gave their informed consent to the use of their biological material to this purpose.

\section{Author Contributions}

EDP, MD, CN, GC and SGP conceived the research and planned the experiments; EDP, FL, MM, JEAC, HH and PP performed the experiments; MD contributes to discussion of the data; EDP, FL, MM, JEAC, MD, CN, GC and SGP discussed and analyzed the data; and EDP, FL, MM, MD, CN, GC and SGP wrote the article.

1. Josowitz R, Carvajal-Vergara X, Lemischka IR, Gelb BD. Induced pluripotent stem cell-derived cardiomyocytes as models for genetic cardiovascular disorders. Curr Opin Cardiol 2011; 26: 223-229.

2. Park $\mathrm{IH}$, Arora $\mathrm{N}$, Huo $\mathrm{H}$, Maherali $\mathrm{N}$, Ahfeldt $\mathrm{T}$, Shimamura A et al. Disease-specific induced pluripotent stem cells. Cell 2008; 134: 877-886.

3. Priori SG, Napolitano C, Di Pasquale E, Condorelli G. Induced pluripotent stem cell-derived cardiomyocytes in studies of inherited arrhythmias. J Clin Invest 2013; 123: 84-91.

4. Carvajal-Vergara X, Sevilla A, D'Souza SL, Ang YS, Schaniel C, Lee DF et al. Patient-specific induced pluripotent stem-cell-derived models of LEOPARD syndrome. Nature 2011; 465: 808-812.

5. Itzhaki I, Maizels L, Huber I, Zwi-Dantsis L, Caspi O, Winterstern A et al. Modelling the long QT syndrome with induced pluripotent stem cells. Nature 2011; 471: 225-229.

6. Jung CB, Moretti A, Mederos y Schnitzler M, lop L, Storch U, Bellin M et al. Dantrolene rescues arrhythmogenic RYR2 defect in a patient-specific stem cell model of catecholaminergic polymorphic ventricular tachycardia. EMBO Mol Med 2012; 4: 180-191.

7. Sun N, Yazawa M, Liu J, Han L, Sanchez-Freire V, Abilez OJ et al. Patient-specific induced pluripotent stem cells as a model for familial dilated cardiomyopathy. Sci Transl Med 2012; 4: 130 ra147.

8. Yazawa M, Hsueh B, Jia X, Pasca AM, Bernstein JA, Hallmayer J et al. Using induced pluripotent stem cells to investigate cardiac phenotypes in Timothy syndrome. Nature 2011; 471: 230-234.

9. Moretti A, Bellin M, Welling A, Jung CB, Lam JT, Bott-Flugel $L$ et al. Patient-specific induced pluripotent stem-cell models for long-QT syndrome. N Engl J Med 2010; 363: 1397-1409. 
10. Itzhaki I, Maizels L, Huber I, Gepstein A, Arbel G, Caspi O et al Modeling of catecholaminergic polymorphic ventricular tachycardia with patient-specific human-induced pluripotent stem cells. J Am Coll Cardiol 2012; 60: 990-1000.

11. Fatima A, Xu G, Shao K, Papadopoulos S, Lehmann M, Arnaiz-Cot JJ et al. In vitro modeling of ryanodine receptor 2 dysfunction using human induced pluripotent stem cells. Cell Physiol Biochem 2011; 28: 579-592.

12. Kujala K, Paavola J, Lahti A, Larsson K, Pekkanen-Mattila M, Viitasalo M et al. Cell model of catecholaminergic polymorphic ventricular tachycardia reveals early and delayed afterdepolarizations. PLoS One 2012; 7: e44660.

13. Novak A, Barad L, Zeevi-Levin N, Shick R, Shtrichman R, Lorber A et al. Cardiomyocytes generated from CPVTD307H patients are arrhythmogenic in response to beta-adrenergic stimulation. J Cell Mol Med 2012; 16: 468-482.

14. Lahat $H$, Pras E, Eldar M. A missense mutation in CASQ2 is associated with autosomal recessive catecholamine-induced polymorphic ventricular tachycardia in Bedouin families from Israel. Ann Med 2004; 36(Suppl 1): 87-91.

15. Priori SG, Napolitano C, Tiso N, Memmi M, Vignati G, Bloise R et al. Mutations in the cardiac ryanodine receptor gene (hRyR2) underlie catecholaminergic polymorphic ventricular tachycardia. Circulation 2001; 103: 196-200.

16. Marx SO, Marks AR. Dysfunctional ryanodine receptors in the heart: new insights into complex cardiovascular diseases. J Mol Cell Cardiol 2013; 58: 225-231.

17. Venetucci L, Denegri M, Napolitano C, Priori SG. Inherited calcium channelopathies in the pathophysiology of arrhythmias. Nat Rev Cardiol 2012; 9: 561-575.

18. Priori SG, Napolitano C, Memmi M, Colombi B, Drago F, Gasparini M et al. Clinical and molecular characterization of patients with catecholaminergic polymorphic ventricula tachycardia. Circulation 2002; 106: 69-74.

19. Cerrone M, Cummings S, Alansari T, Priori SG. A clinical approach to inherited arrhythmias. Circ Cardiovasc Genet 2012; 5: 581-590.

20. Ai X, Curran JW, Shannon TR, Bers DM, Pogwizd SM. $\mathrm{Ca}^{2+} /$ calmodulin-dependent protein kinase modulates cardiac ryanodine receptor phosphorylation and sarcoplasmic reticulum $\mathrm{Ca}^{2+}$ leak in heart failure. Circ Res 2005; 97: 1314-1322.

21. Liu N, Ruan Y, Denegri M, Bachetti T, Li Y, Colombi B et al. Calmodulin kinase II inhibition prevents arrhythmias in RyR2(R4496C $+/-$ ) mice with catecholaminergic polymorphic ventricular tachycardia. J Mol Cell Cardiol 2011; 50: 214-222.

22. Terentyev D, Belevych AE, Terentyeva R, Martin MM, Malana GE, Kuhn DE et al. miR-1 overexpression enhances $\mathrm{Ca}(2+)$ release and promotes cardiac arrhythmogenesis by targeting PP2A regulatory subunit B56alpha and causing CaMKII-dependent hyperphosphorylation of RyR2. Circ Res 2009; 104: 514-521.

23. Di Pasquale E, Belle S, Condorelli G. Generation of human cardiomyocytes: a differentiation protocol from feeder-free human induced pluripotent stem cells. J Vis Exp 2013; 76; doi:10.3791/50429

24. Zhu WZ, Xie Y, Moyes KW, Gold JD, Askari B, Laflamme MA. Neuregulin/ErbB signaling regulates cardiac subtype specification in differentiating human embryonic stem cells. Circ Res 2010; 107: 776-786.

25. Myles RC, Wang L, Kang C, Bers DM, Ripplinger CM. Local beta-adrenergic stimulation overcomes source-sink mismatch to generate focal arrhythmia. Circ Res 2012; 110: 1454-1464.

26. Abriel H, Zaklyazminskaya EV. Cardiac channelopathies: genetic and molecula mechanisms. Gene 2012: 517: 1-11.

27. Priori SG, Chen SR. Inherited dysfunction of sarcoplasmic reticulum $\mathrm{Ca}^{2+}$ handling and arrhythmogenesis. Circ Res 2011; 108: 871-883.

28. Mercola M, Colas A, Willems E. Induced pluripotent stem cells in cardiovascular drug discovery. Circ Res 2013; 112: 534-548.

29. Herron TJ, Lee P, Jalife J. Optical imaging of voltage and calcium in cardiac cells \& tissues. Circ Res 2012; 110: 609-623.
30. Liu N, Napolitano C, Venetucci LA, Priori SG. Flecainide and antiarrhythmic effects in a mouse model of catecholaminergic polymorphic ventricular tachycardia. Trends Cardiovasc Med 2012; 22: 35-39.

31. Watanabe H, Chopra N, Laver D, Hwang HS, Davies SS, Roach DE et al. Flecainide prevents catecholaminergic polymorphic ventricular tachycardia in mice and humans. Nat Med 2009; 15: 380-383.

32. Liu N, Denegri M, Ruan Y, Avelino-Cruz JE, Perissi A, Negri S et al. Short communication: flecainide exerts an antiarrhythmic effect in a mouse model of catecholaminergic polymorphic ventricular tachycardia by increasing the threshold for triggered activity. Circ Res 2011; 109: 291-295.

33. Sikkel MB, Collins TP, Rowlands C, Shah M, O'Gara P, Williams AJ et al. Flecainide reduces $\mathrm{Ca}^{2+}$ spark and wave frequency via inhibition of the sarcolemmal sodium current. Cardiovasc Res 2013; 98: 286-296.

34. Kobayashi S, Yano M, Suetomi T, Ono M, Tateishi H, Mochizuki M et al. Dantrolene, a therapeutic agent for malignant hyperthermia, markedly improves the function of failing cardiomyocytes by stabilizing interdomain interactions within the ryanodine receptor. J Am Coll Cardiol 2009; 53: 1993-2005.

35. Maxwell JT, Domeier TL, Blatter LA. Dantrolene prevents arrhythmogenic $\mathrm{Ca}^{2+}$ release in heart failure. Am J Physiol Heart Circ Physiol 2011; 302: H953-H963.

36. Schindelin J, Arganda-Carreras I, Frise E, Kaynig V, Longair M, Pietzsch T et al. Fiji: an open-source platform for biological-image analysis. Nat Methods 2012; 9 : $676-682$

37. Sullivan S, Egli D, Akutsu H, Melton DA, Eggan K, Cowan C. Derivation of human embryonic stem cells. Hum Embryonic Stem Cells (The Practical Handbook) 2007, chapter 4: pp 35-51.

38. Takahashi K, Tanabe K, Ohnuki M, Narita M, Ichisaka T, Tomoda K et al. Induction of pluripotent stem cells from adult human fibroblasts by defined factors. Cell 2007; 131: 861-872.

39. Yu J, Vodyanik MA, Smuga-Otto K, Antosiewicz-Bourget J, Frane JL, Tian S et al. Induced pluripotent stem cell lines derived from human somatic cells. Science 2007; 318: 1917-1920.

40. Gallo P, Grimaldi S, Latronico MV, Bonci D, Pagliuca A, Gallo P et al. A lentiviral vector with a short troponin-I promoter for tracking cardiomyocyte differentiation of human embryonic stem cells. Gene Ther 2008; 15: 161-170.

41. Huangfu D, Maehr R, Guo W, Eijkelenboom A, Snitow M, Chen AE et al. Induction of pluripotent stem cells by defined factors is greatly improved by small-molecule compounds. Nat Biotechnol 2008; 26: 795-797.

42. Pfaffl MW, Horgan GW, Dempfle L. Relative expression software tool (REST) for group-wise comparison and statistical analysis of relative expression results in real-time PCR. Nucleic Acids Res 2002; 30: e36.

43. Miragoli M, Novak P, Ruenraroengsak P, Shevchuk Al, Korchev YE, Lab MJ et al. Functional interaction between charged nanoparticles and cardiac tissue: a new paradigm for cardiac arrhythmia? Nanomedicine (Lond) 2012; 8 : 725-737.

(1) (2) Cell Death and Disease is an open-access journal published by Nature Publishing Group. This work is licensed under a Creative Commons Attribution-NonCommercialShareAlike 3.0 Unported License. To view a copy of this license, visit http://creativecommons.org/licenses/by-nc-sa/3.0/

Supplementary Information accompanies this paper on Cell Death and Disease website (http://www.nature.com/cddis) 